\title{
Mit dem Klimalotsen Risiken des Klimawandels gezielt umschiffen
}

\author{
Seit Herbst 2010 können Unternehmen ein \\ kostenloses Angebot vom Kompetenzzentrum \\ „Klimafolgen und Anpassung“ des Umweltbun- \\ desamtes nutzen, um sich über Risiken und \\ Chancen des Klimawandels zu informieren. In \\ fünf Schritten unterstützt der Internet-Leitfaden \\ „Klimalotse“ interessierte Nutzer, Bausteine für \\ eine Anpassungsstrategie zu erarbeiten. \\ Von Christian Kind und Till Mohns
}

D er Klimawandel stellt Unternehmen vor neue Herausforderungen. Klimabedingte Risiken werden in den kommenden Jahrzehnten branchenübergreifend zunehmen. Weitere, für die kommenden Jahre zu erwartende Rekordsommer werden beispielsweise mit Niedrigwasser in Flüssen die Verfügbarkeit von Wasser für Kühlprozesse sowie den Schiffstransport beeinträchtigen. In einigen Regionen Deutschlands wird die Intensität von Hagelschauern zunehmen. Damit steigen die Risiken für Schäden an Glasbauten und Gütern, die im Freien gelagert werden.

Neben diesen direkten, physikalisch bedingten Klimarisiken entstehen auch indirekte Risiken, die sich daraus ergeben, dass andere Akteure wie Kunden, Investoren, Versicherer oder Regulierer ihr Verhalten aufgrund des Klimawandels verändern. Dies kann beispielsweise zu Nachfrageveränderungen, höheren Versicherungsprämien oder restriktiveren Berichtspflichten führen. Der Klimawandel kann somit Risiken verstärken oder neu entstehen lassen. Für vorausschauende, innovationsfreudige Organisationen können sich jedoch auch neue Chancen eröffnen. Beides erfordert eine systematische Auseinandersetzung mit Klimafolgen und Anpassung auch in Deutschland.

\section{Barrieren beim Umgang mit Klimarisiken}

Expertise zu Klimawandel und Folgen ist gerade in kleinen und mittelständischen Unternehmen rar. Die verfügbaren Informationen zu Klimaveränderungen sind für Laien oft nicht verständlich. Zudem deckt sich der Planungshorizont von Unternehmen nicht mit den langfristigen Modellen und Projektionen von Klimaforschern.

Folglich werden unternehmerische Entscheidungen häufig weiterhin unter der Annahme getroffen, dass das Klima in Deutschland so bleibt wie bisher. Es besteht die Gefahr, dass die schleichende Zunahme von Risiken, die durch den Klima- wandel entstehen oder verstärkt werden, im Tagesgeschäft und in der langfristigen Planung erst zu spät beachtet werden. Gerade bei strategischen Investitionsentscheidungen kann dies bei voranschreitendem Klimawandel jedoch zu unkalkulierbaren Risiken führen.

\section{Das Konzept des Klimalotsen}

Mit dem Klimalotsen haben adelphi und das Fraunhofer ISI im Auftrag des Umweltbundesamtes für das Kompetenzzentrum Klimafolgen und Anpassung, kurz KomPass, ein neues Informationsangebot konzipiert (1). Hier können sich Entscheidungsträger über die Relevanz von Risiken und Chancen des Klimawandels informieren. Der Klimalotse bietet eine anschauliche Einleitung in das Thema Klimafolgen und Anpassung, für die kein Vorwissen nötig ist. Das Angebot richtet sich sowohl an Unternehmen als auch an Kommunen und enthält für beide Zielgruppen spezifische Informationen und Beispiele. In fünf Schritten können Nutzer(innen) sich mit den zentralen Fragestellungen und Methoden für die Anpassung vertraut machen.

Hierzu werden zu Beginn die bisherigen und die erwarteten Klimaveränderungen in Deutschland vorgestellt. Über eine Darstellung der hieraus resultierenden Auswirkungen für Unternehmen werden Nutzer(innen) zu Methoden für die Analyse möglicher Klimarisiken geführt. Anschließend skizziert der Klimalotse mögliche Vorgehensweisen zur Entwicklung und Bewertung von Anpassungsmaßnahmen. Zum Abschluss des Leitfadens können sich die Nutzer(innen) mit Ansätzen zur Zusammenführung von Maßnahmen zu einer Anpassungsstrategie und mit dem Monitoring der Strategie vertraut machen. Hier hebt der Klimalotse die Bedeutung der Integration von Anpassung in bestehende Unternehmensabläufe und -pläne hervor.

Auch zu Formalisierung und Kommunikation von Anpassungsstrategien gibt der Klimalotse Orientierung. So sollte der Bezug von Anpassung zur vielerorts dringlicheren Herausforderung des Klimaschutzes nicht vergessen und Anpassung klar zu Emissionsminderungsmaßnahmen abgegrenzt werden.

\section{Merkmale des Leitfadens}

Um auch Nutzer(innen) mit wenig Zeit für das Thema anzusprechen, umfasst der Klimalotse einen Schnelldurchlauf, mit dem man sich in einer halben Stunde einen Überblick zu Klimafolgen und Anpassung verschaffen kann. Die Langfassung bietet für jeden Bearbeitungsschritt Aufgaben, die Nutzer(innen) im Verlauf systematisch durcharbeiten können. 
Aus der Bearbeitung der Aufgaben wie beispielsweise der Zusammenstellung bisheriger Beeinträchtigungen des eigenen Unternehmens durch Wetterereignisse, entstehen auf diese Weise sukzessiv Bausteine für eine unternehmensspezifische Anpassungsstrategie. Unterstützt werden Nutzer(innen) hierbei zum einen durch eine Excel-basierte Arbeitsmappe, in der Zwischenergebnisse strukturiert dokumentiert werden können.

Für die Identifikation von Klimarisiken und entsprechende Anpassungsmaßnahmen, zwei zentrale Schritte auf dem Weg zur Anpassungsstrategie, werden zum anderen über eine filterbare Beispielsammlung Anhaltspunkte geboten. Des Weiteren bietet der Klimalotse Literaturverweise sowie Links zu regionalen Klimadaten und weiterführenden Informationsangeboten.

\section{Partizipation im Anpassungsprozess}

Dennoch sind beim Klimalotsen in erster Linie die Nutzer(innen) selbst gefragt. Der Lotse erstellt keine Anpassungsstrategie auf Knopfdruck. Die Gefährdung durch Klimarisiken und die Wirksamkeit von Anpassungsmaßnahmen ist lokal unterschiedlich und stark von der jeweiligen Branche abhängig. Der Klimalotse bietet deshalb vielmehr einen systematischen Rahmen und gibt beispielsweise durch Leitfragen zum Aufsetzen von Anpassungsvorhaben Anregungen, wie Anpassung im Unternehmen erfolgreich gestaltet werden kann.

Klimarisiken betreffen unterschiedliche Unternehmensbereiche. Der Klimalotse liefert Ansatzpunkte für die Verortung der Betroffenheit und schlägt vor, über interne Umfragen und abteilungsübergreifende Workshops betroffene Bereiche möglichst früh in den Prozess einzubeziehen.

Auch für die Umsetzung von Anpassungsmaßnahmen ist Partizipation wichtig, um Handlungsbarrieren zu verringern und mögliche Synergien mit dem Umfeld zu nutzen, beispielsweise in Zusammenarbeit mit Kommunen oder anderen betroffenen Unternehmen. Nicht nur für die Gestaltung partizipativer Prozesse lassen sich hierzu die Vorlagen aus der Arbeitsmappe des Klimalotsen anpassen, um beispielsweise in einer größeren Gruppe Anpassungsmaßnahmen analysieren zu können.

\section{Entscheiden unter Unsicherheit}

Der Klimalotse betont, dass Anpassung an den Klimawandel ein komplexes Vorhaben ist und Unsicherheiten berührt, die sich nur bedingt reduzieren lassen. Aus diesem Grund kommen flexiblen Maßnahmen und dem Monitoring der Strategie eine hohe Bedeutung zu. Ein sprunghaftes Anpassen an einen bestimmten Zustand erscheint weder möglich noch angemessen. Unternehmen sollten hingegen möglichst frühzeitig nachsteuerbare Maßnahmen umsetzen und eine Anpassungsstrategie regelmäßig vor dem Hintergrund neuer Erkenntnisse über Klimarisiken und Anpassungsmaßnahmen aktualisieren.

Der Klimalotse kann dabei unterstützen, die Risiken des Klimawandels zu umschiffen und Chancen gezielt zu verfolgen.

\author{
„Unternehmen sollten möglichst \\ frühzeitig Maßnahmen umsetzen \\ und ihre Anpassungsstrategie \\ regelmäßig aktualisieren."
}

Unternehmen, welche den Herausforderungen des Klimawandels antizipierend und systematisch begegnen, können in $\mathrm{Zu-}$ kunft als klimafreundliche und klimarobuste Organisationen Wettbewerbsvorteile gewinnen.

\section{Anmerkungen}

(1) Der Klimalotse ist kostenfrei nutzbar und erfordert keine Registrierung: http://www.klimalotse.anpassung.net

AUTOREN + KONTAKT

Christian Kind und Till Mohns sind Projektmanager bei adelphi.

Christian Kind, adelphi, Caspar-Theyß-Straße 14a, 14193 Berlin. Tel.: +49 30 8906869-16, E-Mail: kind@adelphi.de, Internet: http://www.adelphi.de

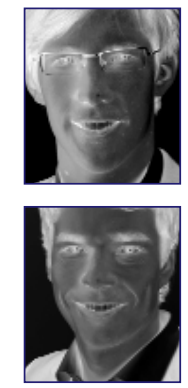




\section{Lizenzhinweis}

Die Beiträge in ÖkologischesWirtschaften werden unter der Creative-Commons-Lizenz "CC 4.0 Attribution Non-Commercial No Derivatives" veröffentlicht. Im Rahmen dieser Lizenz muss der Autor/Urheber stets genannt werden, das Werk darf nicht bearbeitet, abgewandelt oder in anderer Weise verändert und außerdem nicht kommerziell genutzt werden. Die digitale Version des Artikels bleibt für zwei Jahre Abonnent/innen vorbehalten und ist danach im Open Access verfügbar. 